Revista de la red interuniversitaria de estudios sobre las literaturas rioplatenses contemporáneas en Francia

15 | 2016

Un año. Literatura argentina 1969

\title{
Literatura + Teoría = Revolución
}

\section{Graciela Montaldo}

URL: http://journals.openedition.org/lirico/2962

DOI: 10.4000/lirico.2962

ISSN: 2262-8339

Editor

Réseau interuniversitaire d'étude des littératures contemporaines du Río de la Plata

Referencia electrónica

Graciela Montaldo, «Literatura + Teoría = Revolución », Cuadernos LIRICO [En línea], 15 | 2016, Puesto en línea el 05 octubre 2016, consultado el 19 abril 2019. URL : http://journals.openedition.org/ lirico/2962 ; DOI : 10.4000/lirico.2962

Este documento fue generado automáticamente el 19 abril 2019.

\section{(c) $(1) \Theta \Theta$}

Cuadernos LIRICO está distribuido bajo una Licencia Creative Commons Atribución-NoComercialSinDerivar 4.0 Internacional. 


\title{
Literatura + Teoría = Revolución
}

\author{
Graciela Montaldo
}

1 En Las tres fechas César Aira se dedicó a explorar el lado productivo de la literatura pensada en torno a fechas. La crítica literaria deviene así, en sus manos, en el conjunto de reflexiones en torno a la combinación de algunos años relativos a la vida del escritor analizado y a las fechas de publicación de sus obras. Ese lado productivo e instrumental de las fechas no rechaza, sin embargo, la arbitrariedad; por el contrario, la coloca en el centro de nuevas lecturas. Dice Aira: «Cada escritor es un mito, irreductible a los demás; pero la documentación en la que nos llega ese mito está marcada por fechas, que sirven para todos por igual» (2001: 12-13). No voy a reflexionar aquí sobre mitos; cambio los números de la propuesta de Aira porque voy a concentrarme en tres escritores y una sola fecha. La «documentación» en que nos llegan las obras de estos tres escritores coincide en un año y lo que quiero proponer es que la coincidencia nos revela un cambio en la idea de ficción y en la escritura que la lleva a cabo. Ese cambio no «pasó» ese año, pero ese año permite hablar de él.

21969 no solo es un año importante en la cultura argentina. También lo es en términos del diseño de nuevas dimensiones e interrelaciones literarias en América Latina porque marca, en su coincidencia azarosa, un punto de articulación. Como año fetiche, permite repensar cierto giro en la ficción y en la manera de concebir la literatura como institución. Cuando las grandes ficciones del boom ya habían sido consagradas, cuando la literatura latinoamericana había cobrado una dimensión continental e ingresado a la máquina de premios, traducciones, reconocimientos críticos, es decir, cuando se había entrado en una suerte de meseta de productividad estética en donde se garantizaba cierta comodidad con el modelo latinoamericanista (de Julio Cortázar a Gabriel García Márquez, de Mario Vargas Llosa a Carlos Fuentes), el fin de la década introducirá un giro absolutamente nuevo pero que, sin embargo, no irrumpió como una corriente principal sino como una discontinuidad que introdujo cortocircuitos locales, explosiones menores que no intentaron disputar con los exitosos escritores de la época sino poner en cuestión, como otra vanguardia, la institución misma de la escritura, la forma en que se practicaba la literatura. No fue eso lo que hicieron los escritores del boom; por el contrario, con sus 
renovaciones narrativas, fortalecieron como nunca antes la institución literaria. La literatura, como práctica estética, se hizo más y más auto-reflexiva en aquellas ficciones capturando un público que se ampliaba y que disfrutaba y exigía una ficción no del todo realista, con alguna complejidad intelectual y que interpelara los problemas latinoamericanos. Los escritores más vanguardistas de 1969 fueron mucho más allá: hicieron que la literatura se volviera reflexiva de la institución, es decir, la convirtieron en el lugar donde ejercer una reflexión formal y donde estrangular la «naturalidad» con que se estaba experimentando la revolución literaria de los 60 s.

3 Me anticipo a decir que las tres obras publicadas en 1969 que voy a considerar, cuya coincidencia editorial no muestra una correspondencia estética, sí plantean una confluencia en el modo de entender la literatura y en la voluntad de apartarse de los grandes relatos del boom ${ }^{1}$ a la vez que afirman el espacio de la escritura. No las «analizaré»; las tomaré como índice de algo nuevo. Este pequeño corpus se compone de $\mathrm{El}$ apando, novela breve de José Revueltas, publicada en México, y otros dos libros publicados en Buenos Aires, Escrito sobre un cuerpo del cubano Severo Sarduy, y El fiord del argentino Osvaldo Lamborghini. No se parecen entre sí, no plantean los mismos problemas, no pertenecen al mismo género ni se diferencian del mainstream de la época de la misma forma. El libro de Sarduy es un ensayo; los otros dos son ficciones. Lo que permite leer estos textos de manera conjunta es su exploración a veces, compromiso otras, con lo que en términos amplios podríamos llamar «la teoría».

4 Los años 60 trajeron, entre muchas otras cosas, la posibilidad y el desafío de pensar «teóricamente» en todos los ámbitos de la actividad cultural. Entiendo aquí la teoría no como una disciplina o una forma del saber sino como una suerte de dispositivo, como un mandato a enunciar la cultura en términos no solo conceptuales y abstractos sino también creando una red de conexiones entre discursos y prácticas. En esa época la teoría literaria, del arte y del cine, la teoría política, el psicoanálisis, la sociología, la lingüística, comienzan a sofisticar sus categorías, sus argumentos y a interconectarse, a pensarse como campos autónomos pero en diálogo. La teoría fue, para la zona más renovadora del campo intelectual, la nueva vanguardia de los 60; algo que está más allá de las obras, un discurso -cuya articulación se vuelve una nueva práctica- del cual poco a poco será difícil prescindir. La teoría fue la articulación de un discurso que discutiera y volviera a discutir, que cuestionara los presupuestos de las diferentes prácticas culturales y estéticas. Sabemos que el esplendor de la teoría sirvió para transformar la práctica crítica y la reflexión sobre el arte, la literatura, el cine, la música y la cultura en términos amplios; también para darle a las humanidades y a las ciencias sociales un marco reflexivo que habilitó mejores interlocuciones con otras disciplinas y para expandir los objetos de estudio. Pero, más profundamente, fue un pensar, una práctica que sofisticó la producción del arte y generó nuevas identidades intelectuales. Desde entonces la literatura comenzó un proceso de disolución de sus propios límites, un proceso paradójico que la afirmó al tiempo que la hacía estallar por dentro. La literatura comenzó a incorporar, a nivel formal (ya no de contenido) aquello que estaba leyendo en otros discursos, los más sofisticados y renovadores de la época.

Cuando se piensan los grandes nombres de intelectuales de aquellos años, los textos que circulaban entre los grupos intelectuales cada vez más globalizados, aparecen aquellos pensadores nuevos, con saberes y escrituras eclécticas, que introdujeron los cruces de fronteras entre disciplinas: Maurice Blanchot, Roland Barthes, Michel Foucault, Pierre Klossowski, Louis Althusser, Jacques Lacan, entre otros, con sus nuevos usos de los 
campos de saber y los discursos. A lo que se agregan las relecturas de Raymond Roussel, George Bataille y los actualizados pensadores de la Escuela de Frankfurt. El movimiento contrario podría proponerse: que las disciplinas teóricas se «literaturizaron», es decir, se liberaron de la rigidez del formato propiamente disciplinario y entraron en una relación más comprometida con la dimensión estética de la escritura. Eso, claramente, también sucedió, como consecuencia de la centralidad que tomó la «escritura» como tecnología en la transmisión de cualquier discurso. De los posibles movimientos de esos años, me interesa explorar la forma en que la literatura acoge el pensamiento teórico y lo vuelve una productividad estética, quisiera ver cómo los escritores más jóvenes encontraron en la dimensión teórica una forma para renovar una literatura que se sentía cómoda en la repetición de los esquemas que la producían.

\section{La teoría como revolución}

6 Los años en la cárcel fueron intelectualmente productivos para José Revueltas. Su militancia política emancipatoria, bajo el escrutinio de gobiernos represivos, le permitió revertir la parálisis del encierro en proliferación de escrituras. Se diría que las dos principales agencias del intelectual político, la militancia en la calle y a través de la escritura, se articulaban en la secuencia activismo-represión. Al salir de uno de sus encarcelamientos, en 1971, Revueltas escribe un texto en el que sienta las bases de lo que para él debe ser una universidad crítica y autogestionada. Allí afirma, reflexionando sobre los acontecimientos recientes y por los que está confinado, que «el 68 fue un acto teórico» 2. Revueltas se está refiriendo al movimiento estudiantil mexicano y su lucha revolucionaria, del que él fue referente privilegiado como cabeza de la acción emancipadora (y acusado de haber sido el «autor intelectual»). Decir que el 68 fue un acto teórico es haber encontrado una formulación tanto eficaz como seductora; los argumentos serán sólidos y complejos. El 68, por lo demás, no fue un mero acontecimiento. ¿Qué significa entender un evento histórico como acto teórico? Revueltas lo dice de varias maneras, pero lo resume así: «Entendamos aquí el acto teórico como el encuentro de ese tipo de ideas que, al entrar en contacto con una realidad dada, tienen la virtud de remover -trastornar- sus estratos más profundos» (3). El texto apareció publicado pero fue antes una conferencia sobre la universidad autogestionada en el que Revueltas intentó dejar en claro que los actos (hoy, quizás, diríamos «eventos» en términos de Badiou) no están librados a sus propias fuerzas, no son ciegos respecto de sus intenciones sino que, por el contrario, se ligan al mundo de la reflexión, de las ideas, de la abstracción. También se deja en claro que los «actos» son condensaciones de fuerzas históricas que actúan transversalmente. Pero, en realidad, lo que la frase parece revelar también es la precisa y no arbitraria confluencia entre las ideologías y la práctica política (los dos polos más discutidos en la teoría política de los años 60 y los más debatidos entre los intelectuales de izquierda en los años 60 y 70 en América Latina), el modo en que ambas, naturalmente, tendrán que terminar imbricándose en el umbral de la lucha revolucionaria ${ }^{3}$. Revueltas está escribiendo en el aura de los acontecimientos del 68 en México y en todo el mundo, cuando una razón emancipadora se da la mano con una experiencia cuasi-revolucionaria. Las cosas que estaban pasando auguraban cambios radicales que, sin embargo, no vendrían solos: la agencia joven debía estar a la vanguardia y llevar a cabo los actos teóricos que abrieran el camino a la humanidad emancipada. Y el mundo parecía acoger aquella razón de la historia. Por eso sostiene: 
El Movimiento de 1968 fue esencialmente un acto teórico, una acción teórica. ¿Qué se quiere decir con esto y que, por otra parte, tiene una enorme importancia? Ante todo no se trata de desvalorizar la teoría subordinándola al practicismo y al activismo cegatones y vacíos de contenido. Tampoco se trata de afirmar que la teoría se haga en la calle -como piensan algunos- nazca de la turbamulta. [...]. Lo que le da a nuestro Movimiento del 68 la naturaleza de un acto teórico, de una acción teórica, es exactamente la naturaleza misma de la teoría, su propia forma de ser, su razón de ser, su modo de actuar (1).

De algún modo, la palabra «teoría» parece valer, en estos textos, por la palabra «revolución». La sutura entre las dos identidades que la realidad del sistema liberal procuraba mantener separadas se establece a través de la reflexión y de la práctica intelectual pero también de la revolucionaria. Revueltas, militante de la izquierda radical no lleva a cabo solo una acción política continuada ni una teorización política continua. Expulsado del partido comunista mexicano, casi todas sus intervenciones hay que pensarlas por fuera de los dictados ideológicos de un partido y encontrarlas en lo público, espacio privilegiado donde los actos teóricos pueden producirse. Pero lo público no parece ser el único espacio de «libertad». Revueltas es también el escritor que tiene un programa estético.

8 Y en 1969 publicó una novela fundamental, El apando, que explora otra sutura posible, la que existía entre realismo y experimentación. La teoría, el acto teórico, son formulaciones claves de una nueva experiencia de la representación (tanto política como estética) que Revueltas ensaya en sus ficciones. La novela, de trama «simple», trabaja, sin embargo, con la complejidad de la idea de representación. Tres presos rebeldes, recurrentes en la celda de castigo (apando), intentan ingresar droga al penal en combinación con sus mujeres (la madre de uno y las novias de los otros dos). El relato es breve, de un solo párrafo, dándole entrada a las voces «marcadas» de los diferentes personajes. La cárcel no solo es cifra de lo social, es el lugar en donde las tecnologías del poder se ponen en escena, donde las subjetividades sociales actúan. No hay, en esta ficción, ningún intento mimético, ningún juicio moral; hay sí un relato que recoge las tensiones históricas, las pulsiones individuales, las hablas sociales y las expone a una lectura que nos confronta con la objetividad del poder. Los tres prisioneros del relato de Revueltas son pura agencia: pelean, traman, traicionan, recuerdan y, básicamente, desean. El plan de los presos (el complot) sale mal porque hay traiciones entre ellos y porque la institución carcelaria caerá sobre todos: presos en celda de castigo, presos comunes que los apoyan, visitas, guardianes. Los móviles de la trama serán los mecanismos de una sociedad disciplinaria que aún cuando se descuida, aún cuando falla y fracasa en sus intentos de control total, siempre es represiva y violenta con aquellos que están bajo su poder. Una sociedad disciplinaria que, aunque pierda, siempre gana.

\section{Teoría y cuerpos}

Coincidentemente y sin conexiones personales, ese mismo año se publica $E l$ fiord, de Osvaldo Lamborghini. Como si de otro efecto de los actos teóricos se tratara, este relato está ligado a los acontecimientos políticos de la Argentina de fines de los años 60 (no a uno en particular, sino al clima de violencia política, al sentimiento de umbral revolucionario) aunque no de una manera tradicional. También aquí podríamos decir que realismo y experimentación se confrontan para, como en el caso de El apando, producir un nuevo tipo de radicalidad estética. Los saberes e intereses estéticos y políticos de 
Lamborghini probablemente no se comparen con los de Revueltas, mucho más estructurados y específicamente trabajados por la reflexión política. Pero Lamborghini también fue un escritor que encontró en el pensamiento teórico una forma de trabajar la ficción. En su caso no fue primeramente la teoría política sino el psicoanálisis. Lamborghini trabaja, desde el psicoanálisis, la coyuntura política como ficción. La teoría en él no funciona necesariamente como una reflexión sino como una forma de trabajar los textos, como un entramado secreto que se aloja como un parásito que va a erosionar las formas narrativas, las formas de la ficción, la generación de una trama. Su escritura no es teórica en el sentido de Revueltas; sin embargo, Lamborghini tuvo una relación casi intuitiva con la teoría. Más allá de que la tomara en serio o no, se podría decir que estuvo informado por el pensamiento teórico y que su relación con la tradición literaria estuvo mediada por la teoría, especialmente por el discurso psicoanalítico. Él formó parte activa -al menos en la primera época- de la revista Literal, en donde se fundó una nueva idea de literatura ${ }^{4}$. El Fiord es la escritura brutal de esa nueva teoría de la literatura.

También en Buenos Aires se publica el mismo año Escrito sobre un cuerpo, en la editorial Sudamericana, colección Perspectiva. El libro («librito») de Lamborghini lo había publicado una editorial no comercial, Chinatown, y se vendía a escondidas en una librería de la calle Corrientes, tal como reza el mito. El de Sarduy en cambio, sale en edición de la editorial argentina que había publicado Rayuela (1963) y Cien años de soledad (1967), los grandes éxitos de aquella nueva ficción latinoamericana, aunque Sarduy aparecerá en una colección menor. Escrito sobre un cuerpo ocupa un lugar anómalo dentro de los géneros, una suerte de ensayo potente, que abre una lengua completamente nueva, colocando a la literatura en la materialidad del cuerpo, en la vía del grupo Tel Quel, con el que el autor estaba involucrado en su exilio parisien. El texto de Sarduy, literario, fragmentario, crítico, parte de premisas teóricas fuertes, que además plantea de manera contundente:

Aquí una reflexión marginal se impone: de las tres transgresiones del pensamiento que señalaba Bataille (el propio pensamiento, el erotismo y la muerte), creo que solo una, la primera, subsiste en toda su fuerza. La sociedad burguesa (sobre todo la que no se confiesa serlo) ha mitigado la resistencia que le inspiraban el erotismo y la muerte para intensificar, hasta lo patológico, la que le inspira el pensamiento que se piensa a sí mismo. Blasfemia, homosexualidad, incesto, sadismo, masoquismo y muerte son ya transgresiones relativamente toleradas. (No hablo de la transgresión pueril que es el arte «de denuncia»: el pensamiento burgués no solo no se molesta, sino que se satisface ante la representación de la burguesía como explotación, del capitalismo como podredumbre). Lo único que la burguesía no soporta, lo que la «saca de quicio», es la idea de que el pensamiento pueda pensar sobre el pensamiento, de que el lenguaje pueda hablar del lenguaje, de que un autor no escriba sobre algo sino que escriba algo (como proponía Joyce). Frente a esta transgresión, que era para Bataille el sentido del despertar, se encuentran, repentina y definitivamente de acuerdo, creyentes y ateos, capitalistas y comunistas, aristócratas y proletarios, lectores de Mauriac y de Sartre. La desconfianza y la agresividad que suscitan las búsquedas críticas actuales ilustran la unidad de las ideologías más opuestas ante la verdad del despertar de Bataille (19-20).

11 Recordemos que Sarduy está comenzando su libro, hablando de la secuencia de representación de la violencia que, partiendo de Bataille, va a leer en las obras de Giancarlo Marmori, Salvador Elizondo, Julio Cortázar, Carlos Fuentes, José Donoso. Se trata de una «reflexión marginal» que sustentará, sin embargo, la teoría de todo el libro y que puntúa el centro del problema. Porque ¿qué cosa es la teoría sino ese pensar sobre sí misma? ¿qué es sino esa reflexión del pensamiento sobre sí mismo? Sarduy explorará en 
todo el libro las formas de una violencia social que es también la violencia que la literatura, como práctica política, trae al mundo.

Para hablar de teoría habría que colocarse en el espacio de colisión de los diferentes discursos que durante el siglo XX reorganizaron el pensamiento crítico, es decir, en la intersección entre marxismo, política, psicoanálisis y vanguardia estética. De la alianza crítica entre todas estas experiencias radicales surgieron los discursos teóricos del siglo $\mathrm{XX}$. Lo que hoy llamamos teoría, como discurso autónomo, surgió de esa encrucijada y fue ocupando diferentes lugares. Como todos esos otros discursos, resultó peligrosa desde su aparición porque se preguntaba (y pregunta) por ciertas inmaterialidades y por el constante corrimiento respecto del sentido; la teoría, sin embargo, no ocupa un espacio vacío sino que se interroga por el lugar de la práctica sobre la que intenta reflexionar. A la teoría, como constante problematización del lugar del discurso, se le opone la profesionalización de la actividad literaria y crítica, como a las vanguardias el museo. Se oponen, pero, al mismo tiempo, no vive una sin la otra.

\section{Teoría y lenguaje social}

No resulta extraña esta coincidencia, que abría una nueva línea de experimentación escrituraria. El contexto de «la teoría» estaba muy presente en la Argentina de entonces. David Viñas, en la Argentina de mediados de los 50 (que salía de la experiencia del peronismo, que había cambiado radicalmente los pactos sociales del país liberal) leía «teoría»; leía a Hegel, leía a Marx, leía a Sartre, leía a Lucien Goldman, leía a Arnold Hauser, leía a Louis Althusser, entre muchos otros ${ }^{5}$. Viñas introdujo, junto con sus compañeros de la revista de literatura y política Contorno (1953-1959), una nueva manera de articular diferentes superficies culturales en la que el uso de la teoría no solo era un medio de autorización cultural sino una forma de poner en diálogo el archivo nacional con las discusiones de la izquierda internacional. De algún modo, lo que los jóvenes de entonces buscaban estaba cruzado por esa tensión entre los discursos emancipatorios que se producían en las metrópolis y los nuevos eventos emancipatorios que florecían en lo que entonces se llamaba el «Tercer Mundo». No se trataba de una búsqueda celebratoria de la autenticidad de la periferia, del descubrimiento del margen. Por el contrario, a un lado de la ficción del boom, estos textos buscaban sobre todo una articulación, la posibilidad de crear una interlocución con la comunidad intelectual internacional.

Muchos años después de su circulación, cuando las lecturas de Contorno se hubieron canonizado, uno de los jóvenes intelectuales argentinos que comenzó su carrera en la revista que habían fundado los hermanos David e Ismael Viñas, escribe un libro para revisar su pasado intelectual. Se trata de Carlos Correas, que en 1991 publica La Operación Masotta. Oscar Masotta fue otro intelectual argentino, un poco más joven que quienes integraban el grupo, que llegó a publicar en Contorno y que rápidamente, en el contexto de modernización intelectual y politización profunda de los años 60 , se convirtió en una suerte de estrella que introdujo buena parte de las novedades intelectuales del periodo (el happening y su teoría del arte, el Lacanismo) ${ }^{6}$. Correas escribe su libro cuando ya Masotta ha muerto y lo dedica a hacer una crítica radical a los intelectuales de su generación (la de Viñas, la de Oscar Masotta) afirmando, entre otras cosas, que

¿Qué es la literatura? de Sartre, en su primera edición castellana de 1950, fue nuestro canon. A medias entrevisto (o, si el lector medio lo prefiere: «investigado»), lo recorríamos para extraer palabras y frases para las polémicas y para nuestros 
primeros escritos críticos. Aceptábamos o, mejor, ignorábamos las calidades de la traductora Aurora Bernárdez. Años más tarde, cotejando con el original francés, noté los errores y las incurias de Aurora Bernárdez. Nuestro texto sagrado nos había resultado una parca estafa que nos infligía la negligencia de la editorial Losada. Aquí esta forma de horror argentino nos apresó -e infectó- sin que fuéramos concientes de ello.

Lecturas tronchadas, malentendidas, embaucadoras, ideas apenas sospechadas, alusiones y referencias incomprensibles por falta de contexto, intuiciones aproximativas y sin interés, iluminaciones anticuadas o caducas... no sólo provocaban las consabidas insolvencia o impostura; nos daban también, para satisfacer nuestras pulsiones belicosas, consignas puramente episódicas (24-25). viajeras $»^{7}$ sino incluso de las malas traducciones. Poniendo entre paréntesis sus juicios de valor, la declaración muestra el modo de formación intelectual de una generación para la cual aprender el lenguaje teórico fue decisivo y parte de la identidad intelectual. Leer, estudiar, «investigar», los textos teóricos era aprender a hablar. Un aprendizaje que pudo darse -según Correas- aún en contra de la lógica que lo construía, hecho sin instrumentos adecuados, en desventaja y con un claro desdén por el cuidado intelectual. Hoy diríamos que esa generación, que se formó venerando -sin saberlo- las formas espurias de transmisión cultural de la periferia, quizás no aprendió la exactitud del pensamiento de Sartre (si tal cosa hubiese sido posible) pero sí supo poner en escena los nuevos valores del mundo intelectual que se abría en los años 60. Correas le pasa factura a su generación en lo que tenía de devoción europeísta y, para hacerlo, se pone del lado del purismo. En negativo, se puede leer en su discurso un uso de la teoría como parte indispensable de la nueva identidad intelectual, la búsqueda de los instrumentos, las armas, para defenderse (y abrir batalla) en el tradicional campo literario argentino de aquellos años. La teoría claramente se percibía como el arma para ingresar al campo intelectual ${ }^{8}$.

Las teorías, entonces, no son solo sistemas, categorías, conceptos; son también posiciones, como señala Rancière al describir el saber, que «no es un conjunto de conocimientos, sino una posición» (16). Ocupar un lugar, tratar de colonizar el campo intelectual de sus contemporáneos, fue lo que hizo de las lecturas de Contorno, Viñas, Masotta, una intervención exitosa. La ligereza del aprendizaje, que Correas denuncia con saña, forma parte de la difusión de la teoría: textos que llevan a la discusión, que componen un tipo de intelectual combativo, no necesariamente académico, pero que crea la posición de poder/ saber a través de la cual se postulan nuevas formas de conocimiento. Durante esos años se creó la figura de un intelectual que dispara citas en la mesa de café, que discute la realidad desde los libros, que se apertrecha de un vocabulario y de un sistema de argumentación en los autores «teóricos» que las instituciones rechazan. Masotta, por su parte, a quien Correas pone como paradigma de intelectual con saberes aproximativos, mal comprendidos, poco serios, fue quien difundió la «teoría» (de los medios, del pop-art, de Lacan) en la Argentina, en famosos grupos de estudio que él dirigía, como introductor de la realidad teórica en la Argentina (en su exilio en Barcelona, siguió enseñando «teoría» a los españoles también bajo la modalidad de los grupos de estudio privados). Viñas y Masotta -con pocos pero decisivos años de diferencia- estuvieron entre los primeros intelectuales «teóricos» (interesados en pensar la teoría) de la Argentina de los 50 y $60 \mathrm{~s}$ y quienes desarrollaron nuevas lecturas de la realidad nacional ${ }^{9}$. Con las universidades intervenidas después de cada golpe militar $(1966,1976)$ la principal institución que les hubiese dado cabida a estos intelectuales, los expulsaba a la calle, adonde trasladaron sus saberes y los diseminaron entre compañeros y discípulos jóvenes 
que encontraron un método de trabajo en la informalidad de los grupos de estudio, las librerías o las mesas de cafés. Pero el deseo teórico había infiltrado muchas capas del campo intelectual argentino y los más jóvenes supieron aprovecharse de ese deseo. En la Argentina, Osvaldo Lamborghini fue uno de ellos, pero también Germán García, Luis Gusman y un grupo de escritores que encontraron en la teoría formas de resignificar la ficción, de repolitizar la estética y de cuestionar la institución literaria en su conjunto.

\section{El tiempo en el tiempo}

El año 1969 puede marcar el momento en que una experiencia difusa encuentra formas de articulación potentes. Lo que las obras de Revueltas, Lamborghini y Sarduy vienen a hacer visible es que, en esos años, la literatura -la escritura- se hizo vanguardia, se convirtió en vanguardia política porque disolvió la práctica esteticista y la convirtió en otra cosa, algo que rozó la ilegalidad, que se puso fuera de la ley, que desafió a la institución literaria. Esos textos siguen perteneciendo a una vanguardia irreductible, siguen "sacando de quicio" ya no solo a la burguesía sino a cualquier lector/a. Ciertos márgenes del campo intelectual intentaron colonizarlos con interpretaciones, pero ellos siempre se colocan un poco más allá. Por eso sería posible pensarlos como un momento revolucionario o, más precisamente, como textos-revolución, porque marcan un momento de disolución del orden y la imposibilidad de entrar en otro orden. Marcan el momento en que un libro se vuelve una acción, un hecho que produce no solo sentidos sino que tiene consecuencias políticas (en las políticas de la literatura). Son textos que parecen haber creado un tiempo en que la acción revolucionaria se dispara y que se reactualiza en cada lectura ya no como recreación de un sentido sino de una acción que se sigue desplegando en el tiempo. En ellos la literatura deja de ser el problema de un escritor para volverse problema de todos: es un problema que la literatura esté produciendo estos textos. Los «actos teóricos» no han cesado en el mundo, pero la ola anti-intelectual que trajo el neoliberalismo ha querido desdibujarlos; la reactivación del pensamiento crítico en la última década permite reconsiderarlos aun cuando sea -felizmente- incapaz de condensarlos en el sentido. No parece haber crítica posible de aquellas obras que son a la vez literatura y acto.

\section{BIBLIOGRAFÍA}

Aira, César, Las tres fechas. Rosario: Beatriz Viterbo, 2001.

Avelar, Idelber, Alegorías de la derrota: la ficción postdictatorial y el trabajo del duelo. Santiago de Chile: Cuarto Propio, 2000.

Badiou, Alain, L'être et l'événement. París: Seuil, 2006.

Brizuela, Natalia y Juan Pablo Dabove (eds), Y todo el resto es literatura: ensayos sobre Osvaldo Lamborghini. Buenos Aires: Interzona, 2008. 
Correas, Carlos, La Operación Masotta (cuando la muerte también fracasa). Buenos Aires: Catálogos, 1991.

Draper, Susana, «Experiments in Freedom and Cognitive Democracy in 1968 Mexico» paper presented at «1968 in Latin America: A Roundtable», Columbia University, Institute of Latin American Studies. April 5, 2013.

Forest, Philippe, De Tel Quel à L'Infini: nouveaux essais. Nantes: Cécile Defaut, 2006.

Gilman, Claudia, Entre la pluma y el fusil. Debates y dilemas del escritor revolucionario en América Latina . Buenos Aires: Siglo XXI, 2003.

Lamborghini, Osvaldo, El fiord. Buenos Aires: Chinatown, 1969.

Longoni, Ana y Mestman, Mariano, Del Di Tella a «Tucumán Arde»: vanguardia artística y politica en el '68 argentino. Buenos Aires: Eudeba, 2010.

Mendoza, Juan, «El proyecto Literal», Literal, edición facsimilar. Buenos Aires: Biblioteca Nacional, 2011.

Moreiras, Alberto, Tercer espacio: literatura y duelo en América Latina. Santiago de Chile: Lom, 1999.

Masotta, Oscar, Conciencia y estructura. Buenos Aires: Jorge Álvarez, 1968.

Rancière, Jacques, El espectador emancipado. Buenos Aires: Manantial, 2010.

Revueltas, José, El apando. México: Era, 1969.

¿Qué es la autogestión académica? En línea: http://biblioteca.clacso.edu.ar/ar/libros/

osal/osal24/08revuel2.pdf . Consulta: 15/06/2016.

Said, Edward W., The World, the Text, and the Critic. Cambridge, Massachusetts: Harvard University Press, 1983.

Sarduy, Severo, Escrito sobre un cuerpo. Buenos Aires: Sudamericana, 1969.

Strafacce, Ricardo, Osvaldo Lamborghini, una biografía. Buenos Aires: Mansalva, 2009.

Viñas, David, Literatura argentina y realidad política. Buenos Aires: Jorge Álvarez, 1967.

\section{NOTAS}

1. Los trabajos ya clásicos de Alberto Moreiras (El tercer espacio) e Idelber Avelar (Alegorías de la derrota) hicieron la crítica a la literatura del boom y el realismo mágico y establecieron su lugar en el imaginario latinoamericano.

2. Susana Draper ha estudiado este texto en «Experiments in Freedom and Cognitive Democracy in 1968 Mexico» (2013).

3. Claudia Gilman (2003) estudió la confrontación de las dos identidades en conflicto entre los intelectuales de los años 60.

4. Detalles sobre el grupo y la idelogía literaria de la revista se encuentran en el estudio preliminar de Juan Mendoza (2011) a la edición facsimilar de Literal.

5. Y leía, al mismo tiempo, los archivos de la nación y el canon de la Argentina.

6. Anoto aquí algunos de sus títulos: Happening (1967), Conciencia y estructura (1968); Introducción a la lectura de Jacques Lacan (1977), Ensayos lacanianos (1977), El modelo pulsional (1980), Lecciones de psicoanálisis (1982). 
7. Edward W. Said problematizó las reapropiaciones de la teoría durante el siglo XX, especialmente durante los años 60 , en las zonas periféricas de las principales «usinas filosóficas» europeas.

8. La editorial venezolana Monte Ávila publica Intervenciones. Nueve modelos de crítica, de T. W. Adorno, en 1969, dentro de lo que será un clara política editorial por traducir «teoría» europea desde fines de los años $60 \mathrm{y}$ durante gran parte de los 70 .

9. José Aricó, Oscar del Barco, Héctor Schmucler, y Samuel Kiczkowski, expulsados del Partido Comunista argentino, fundaron en Córdoba la revista Pasado y Presente. Entre los años 1963 y 1965, no serán ajenos a la novedad del pensamiento teórico político, y explorarán la teoría política no solo desde Marx sino desde el pensamiento de Antonio Gramsci.

\section{RESÚMENES}

A partir de tres libros publicados en 1969 (El Apando de José Revueltas, El Fiord de Osvaldo Lamborghini y Escrito sobre un cuerpo de Severo Sarduy), este artículo explora los radicales cambios estéticos que se producen a fines de los años 60 en América Latina. Sostiene que la literatura del boom normaliza un tipo de ficción que interpela a un público ampliado y exhibe renovaciones estéticas que fueron consideradas como radicales. Frente a ese panorama, los tres textos estudiados, proponen una radicalización de la práctica estética que impugna y cuestiona la institución literaria. Lo hacen a través del recurso a la «teoría». Por tal entendemos no una disciplina o una forma del saber sino una suerte de dispositivo, una forma de enunciar la cultura en términos no solo conceptuales y abstractos sino también creando una red de conexiones entre discursos y prácticas. Teoría y radicalidad estética se entrecruzan en las obra de estos tres escritores y crean formas alternativas y radicales de pensar la relación entre literatura y política.

À partir de trois livres publiés en 1969 (El Apando de José Revueltas, El Fiord de Osvaldo Lamborghini et Escrito sobre un cuerpo de Severo Sarduy), cet article explore la radicalité des tournures esthétiques qui se sont produit en Amérique Latine à la fin des années 1960. On soutien que la littérature du boom normalise un type de fiction interpelant un public élargi, au même temps elle porte des innovations esthétiques qui ont été considérées comme radicales. Face à ce contexte, le trois textes étudiés, proposent une radicalisation de la pratique esthétique qui conteste et met en question l'institution littéraire. Ils le font à travers d'un appel à la " théorie». La théorie n'est pas une discipline ou un forme du savoir, mais une sorte de dispositif, une forme de dire la culture en termes pas seulement conceptuels et abstraits, mais aussi en créant un réseau de liens entre pratiques et discours. Théorie et radicalité esthétique s'entrecroisent dans les œuvres de ces trois écrivains et créent formes alternatives et radicales de penser le rapport entre littérature et politique.

This article is focused on three books published in 1969, El Apando by José Revueltas, El Fiord by Osvaldo Lamborghini, and Escrito sobre un cuerpo by Severo Sarduy. It explores the radical aesthetic changes produced in the late $60 \mathrm{~s}$ in Latin America. It argues that the "boom" phenomena produce a literature that becomes the literary norm, interpellates a great audience and develops aesthetic procedures that seemed radical. In this context, the three texts suggest a radicalization of the aesthetic practice that questions literary institution. They do that appealing to "theory." The theory is not a discipline, or a way of knowledge either but a kind of device, a 
way of state the culture in conceptual and abstract terms. But it is also a way of creating a network of connections between discourses and practices. Theory and aesthetic radicalism are interconnected in the works of these three writers. They create an alternative and radical form of thinking the relationship between literature and politics.

ÍNDICE

Mots-clés: Littérature Latino-américaine, culture et politique, Osvaldo Lamborghini, José Revueltas, Severo Sarduy.

Keywords: Latin American Literature, culture and politics

Palabras claves: Literatura latinomericana, cultura y política

\section{AUTOR}

\section{GRACIELA MONTALDO}

Columbia University

gmontaldo@gmail.com 